\title{
Damage Identification of Plate Structure Based on the Method of Modal Flexibility Curvature Difference
}

\author{
Jiangang Zhao ${ }^{1}$, Yuxiang Zhang ${ }^{1}$, and Jiazhao Chen $^{2}$ \\ ${ }^{1}$ Xi' an Research Inst. of Hi-Tech., Shanxi Xi'an, P. R. China \\ ${ }^{2}$ Xi'an Research Inst. of Hi-Tech., Shanxi Xi'an, P. R. China
}

\begin{abstract}
According to the higher order modes of the structure are difficult to extract and the lower order are easy to obtain in practice, it is put forward that only uses the parameters of lower or first-order modal to constitute the modal flexibility curvature difference (MFCD) as the index of damage identification, which based on the flexibility matrix of structure being sensitive to the structure modal characteristics. Numerical analysis is made on different damage conditions of a plate structure, and the result shows that the MFCD can not only accurately identify the single-damage and multi-damage position of plate structure, but also reflect the size of damage degree. It has a great significance to apply the index into the range of damage identification of actual structures.
\end{abstract}

\section{Introduction}

In recent years, the technology of damage identification based on structural vibration is becoming a hot topic. It is found that the damage of structure will generally cause the change of its stiffness, which will change the modal parameters (natural frequency, modal vibration mode and damping) of the structure. Based on the above understanding, some scholars used the frequency and the mode of structural vibration signal to identify the damage early[1-4]. But the frequency and vibration mode are not sensitive to the structural damage, the identification index which is more sensitive to damage is proposed later, such as the modal strain energy method[5], the residual force vector method[6], the flexibility matrix method[7] and so on. Among these indexes, the flexibility matrix method can get accurate damage identification results only need lower order modal parameters, so it has more practical application value (Because the higher order modes of the structure are difficult to extract and the lower order are easy to obtain in practice). According to the study, the flexure value of the structure changes greatly in the damaged position or the adjacent area, and the flexure value changes differently with different degree of damage[8]. By comparing several methods of modal flexibility matrix which are used to identify the structure damage, it is found that [9-14] whatever damage, only the MFCD can clearly indicate the location of the single-damage and multi-damage, and can reflect the size of damage degree, the effect is better than others. Most of the above researches are based on the damage identification of beam structures, but few studies have been done on the application of the MFCD to plate structures or shell structures.
In this paper, the MFCD is proposed to identify the plate damage, and numerical analysis is made on different damage conditions of a plate structure. Firstly, according to the theory of Kirchhof thin plate, the plate structure is divided and the flexibility matrix of structure response is constructed. Secondly, the change of flexibility value is amplified by the second order differential, and the difference of flexibility curvature matrix which are before and after structural damage is further obtained. Finally, through numerical calculation, it is shown that the damage can be accurately located only with lower order or first-order modal parameters, and the size of damage degree can be effectively determined. It has a great significance to apply the index into the range of damage identification of actual structures.

\section{Fundamental}

According to the modal parameters of plate structure, the flexibility matrix of structure is:

$$
F=\sum_{i=1}^{r} \frac{1}{\omega_{i}^{2}} \varphi_{i} \cdot \varphi_{i}^{T}
$$

Where, $\omega_{i}$ is the natural frequency of the structure, and $\varphi_{i}$ is the vibration mode after normalization of the structure mass. We can see from formula (1), the structure of the flexibility matrix $F$ is inversely proportional to the square of $\omega_{i}$, and is proportional to the square of $\varphi_{i}$, it indicates that $F$ is more sensitive to natural frequency and modal mode. The plate structure is divided into $(a-1) \times(b-1)$ units according to the 
assumption of Kirchhoff thin plate, Let the mode or rotation vector of the $\mathrm{i}^{\text {th }}$ order be:

$$
\begin{aligned}
& \varphi_{i}=\left[a_{i 1}, \cdots, a_{i j}, \cdots, a_{i n}\right]_{n \times 1}^{T} \\
& (i=1,2, \cdots, r ; j=1,2, \cdots, n),
\end{aligned}
$$

Where: $a_{i j}$--the displacement or rotation value of the node in column $j$ of row $i, n=a \times b ; r$ is the total number of modes, and $n$ is the number of nodes.

We can get the transition matrix $F_{t a}$ from formula (1):

$$
F_{t a}=\left[\begin{array}{ccc}
\sum_{i=1}^{r} \frac{1}{\omega_{i}^{2}} a_{i 1} \cdot a_{i 1} & \ldots & \sum_{i=1}^{r} \frac{1}{\omega_{i}^{2}} a_{i 1} \cdot a_{i n} \\
\vdots & \ddots & \vdots \\
\sum_{i=1}^{r} \frac{1}{\omega_{i}^{2}} a_{i n} \cdot a_{i 1} & \ldots & \sum_{i=1}^{r} \frac{1}{\omega_{i}^{2}} a_{i n} \cdot a_{i n}
\end{array}\right]
$$

Let $C_{k}$ be the maximum value of each column element in the above matrix, so we get the transition matrix $F_{t b}$ :

$$
F_{t b}=\left[C_{1}, \cdots, C_{k}, \cdots, C_{n}\right]_{1 \times n}
$$

The maximum flexibility matrix is constructed from $F_{t b}$ to $F_{p}$ :

$$
F_{p}=\left[\begin{array}{ccc}
C_{1} & \cdots & C_{b} \\
\vdots & & \vdots \\
C_{(l-1) b+1} & & C_{(l-1) b} \\
\vdots & & \vdots \\
C_{(a-1) b+1} & \cdots & C_{n}
\end{array}\right]_{a \times b}
$$

For each element in $F_{p}$ of the plate structure, it corresponds to the $z$ direction displacement and $x, y$ direction rotation angle of each node, that is, each row or column in the matrix is the flexibility of the coordinate function. Near the point of structural damage, the flexibility will change, and this change will become more pronounced with the action of the second derivative.

The transverse flexibility curvature matrix $F_{c h}$ of the structure is:

$$
F_{c h}=\frac{1}{S} F_{c t h} F_{p}
$$

Where:

$$
F_{c t h}=\left[\begin{array}{cc}
0 & E_{(a-1) \times(a-1)} \\
0 & 0
\end{array}\right]_{a \times a}-2 E_{a \times a}+\left[\begin{array}{cc}
0 & E_{(a-1) \times(a-1)} \\
0 & 0
\end{array}\right]_{a \times a}^{T}
$$

Among them: $E_{(a-1) \times(a-1)}{ }^{--} \operatorname{Order}(a-1)$ unit matrix;

$E_{a \times a}$-- Order $a$ unit matrix;

$S$--The unit area.
From the above, the flexural curvature difference between before and after structural damage is obtained, that is, the transverse damage index $D_{h}$ (MFCD):

$$
D_{h}=F_{c h w}-F_{c h d}
$$

Among them: $F_{c h w}$ is the transverse flexibility curvature of intact structure, $F_{c h d}$ is the transverse flexibility curvature of damage structure. It can be seen from the above that the damage index after the second order differential is amplified and more sensitive to modal parameters.

The longitudinal flexibility curvature matrix $F_{c v}$ of the structure is:

$$
F_{c v}=\frac{1}{S}\left[F_{c t v} F_{p}^{T}\right]^{T}
$$

Where: $E_{(b-1) \times(b-1)}$-- Order $(b-1)$ unit matrix; $E_{b \times b}--$ Order $b$ unit matrix.

The flexural curvature difference before and after structural damage is obtained, that is, the longitudinal damage index $D_{v}$ (MFCD):

$$
D_{v}=F_{c v w}-F_{c v d}
$$

Among them: $F_{c v w}$ is the longitudinal flexibility curvature of intact structure, $F_{c v d}$ is the longitudinal flexibility curvature of damage structure.

According to the difference of modal flexibility curvature matrix of the damaged structure, the $3 \mathrm{D}$ image of damage index is drawn. At the same time, the size of damage degree can be determined by comparing the damage index value at the node of damaged unit.

\section{Research on damage identification of plate structure}

\subsection{The finite element modal of plate structure}

Considering a square aluminum alloy plate with four fixed corners (The length of side is $1.0 \mathrm{~m}$, the thickness is $0.005 \mathrm{~m}$ ), and the finite element modal is shown in Fig. 1. 


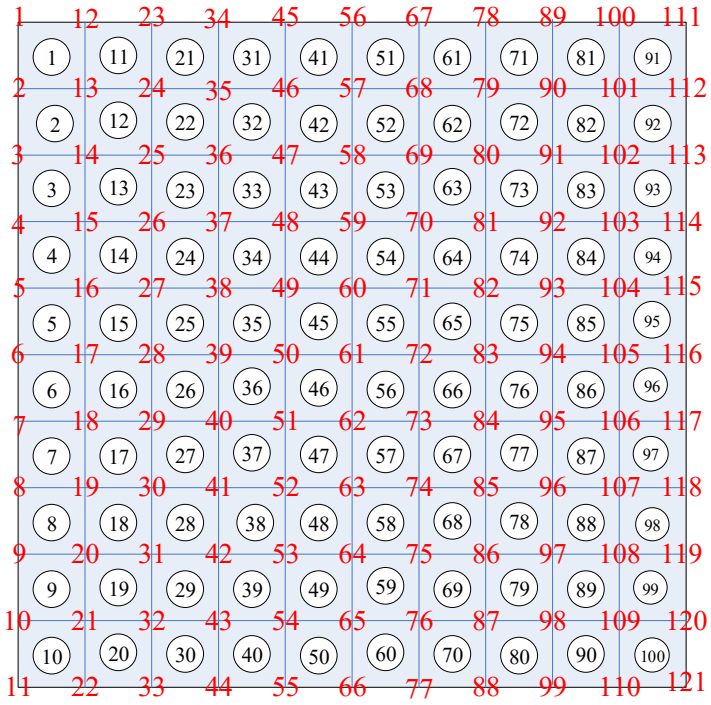

Fig.1. The finite element modal of plate structure.

Where: Elastic modulus of material $E=73 G P a$, Poisson's ratio $\mu=0.33$, the density of material $\rho=2870 \mathrm{Kg} / \mathrm{m}^{3}$. In this paper, the damage is simulated by reducing the elastic modulus of the structural element; the size of element is $0.1 m \times 0.1 m$.

In the finite processing[15], the following matrix representation is applied relation to the node number in the figure:

$$
\left[\begin{array}{ccc}
111 & \cdots & 121 \\
\vdots & & \vdots \\
1 & \cdots & 11
\end{array}\right]
$$

As opposed to the unit number in the diagram, the following matrix representation is applied:

$$
\left[\begin{array}{ccc}
90 & \cdots & 100 \\
\vdots & & \vdots \\
1 & \cdots & 10
\end{array}\right]
$$

In the processing of dealing with the matrix of flexibility maximum value and the flexibility curvature matrix difference that is damage index matrix, the following matrix is arranged (I'm just going to give you the horizontal direction, so you get the vertical direction):

$$
\left[\begin{array}{ccc}
11_{1} & \cdots & 11_{11} \\
\vdots & a_{i} & \vdots \\
1_{1} & \cdots & 1_{11}
\end{array}\right]
$$

$a$ in $a_{i}$ is the number of rows, and $i$ is the number of nodes in each row. For example, the damage unit node number of the corresponding finite element are $72,73,83$, 84 and the unit number is 66 . The corresponding damage index matrix are number 6,7 nodes in line 7 and 6,7 nodes in line 8 .

\subsection{Damage analysis}

In this paper, we take the reduction of element stiffness to simulate the damage of structure, and divide the damage into single-position damage and multi-position damage. The identification of single damage is analyzed by unit 45 damaging in the middle of the plate or unit 95 damaging at the edge of plate respectively. Multi-position damage identification is divided into unit 45 and unit 95 in the same row or unit 23 and unit 77 in different columns in different rows. We analyze the unit 45 damage $20 \%, 50 \%, 70 \%$ respectively to judge the degree of damage and compare the damage degree of multi-position damage. The details is in table 1.

Table 1. The table of structural damage condition.

\begin{tabular}{c|c|c}
\hline $\begin{array}{c}\text { Damage } \\
\text { condition }\end{array}$ & $\begin{array}{c}\text { Damage } \\
\text { element }\end{array}$ & The size of damage degree \\
\hline Case1 & 45 & Be damaged by $50 \%$ \\
Case 2 & 95 & $\begin{array}{c}\text { Be damaged by } 50 \% \\
\text { Both are damaged by } 50 \%\end{array}$ \\
Case 3 & 45,95 & $\begin{array}{c}\text { Both are damaged by } 20 \% \\
\text { Case 4 }\end{array}$ \\
Case 5 & 45,77 & $\begin{array}{c}\text { Be damaged by } 20 \%, 50 \%, 70 \% \\
\text { respectively }\end{array}$ \\
Case 6 & 23,77 & $\begin{array}{c}\text { Unit 23 is damaged by } 20 \% ; \text { Unit } \\
77 \text { is damaged by } 70 \%\end{array}$ \\
\hline \hline
\end{tabular}

\subsection{Numerical analysis}

When it comes to numerical calculation, you need to get the modal information of the structure to extract $\omega_{i}$ which is the modal frequency and $\phi_{i}$ which is the corresponding displacement or corner vector, and then to deal with $\phi_{i}$ to obtain $\varphi_{i}$ that is the displacement or rotation vector of the mass normalization. We take the transverse flexibility curvature difference as damage index in this paper, and then extract the frequency and displacement vector of foregoing three orders modal, numerical analysis is as follows:

\subsubsection{Single-position damage identification}

We have calculated the damage index of the first order, that was shown in Fig 2, and the damage index of foregoing three orders, that was shown in Fig 3, that the unit 45 in the middle of the plate is damaged by $50 \%$.

The Fig 2 is the figure of the first order damage index when the unit 45 in the middle of the square aluminum alloy plate is damaged by $50 \%$. Among them, Fig. 2 (a) is a three-dimensional view of the damage index, from which it can be clearly seen that unit 45 has damage; In order to further determine the damage location, the section curves of the $5^{\text {th }}$ row and $6^{\text {th }}$ row of Fig. 2(a) are drawn, as shown in Fig. 2(b). We can see obviously from Fig. 2(b), the damage indexes all have a step at the position of node 5 and node 6 which are belong to $5^{\text {th }}$ 
curve and $6^{\text {th }}$ curve, exactly corresponding to the node 49 , node 50, node 60 and node 61 in the finite element structure, that are the four nodes of the unit 45 . So we can sure that the unit 45 has damage.

The Fig. 3 is the figure of the foregoing three orders damage index when the unit 45 in the middle of the square aluminum alloy plate is damaged by $50 \%$. Similar to the above analysis, the damage of unit 45 was determined. Comparing Fig.2 and Fig.3, it can be seen that there are more peak areas in the $3 \mathrm{D}$ view of the foregoing three orders damage index. If the damage occurs in the area where the peak belongs to, it is easy to cause identification error. Therefore, the damage position can be determined by using the modal frequency of the first order and the corresponding displacement vector based on the damage identification index of flexibility curvature difference. In the analysis below, we only use the index obtained from the first order modal parameters to identify the damage.

The first order damage index of unit 95 at the edge of the plate is calculated when the damage is $50 \%$, as shown in Fig. 4.

The Fig. 4 is the figure of the first order damage index when the unit 95 at the edge of the square aluminum alloy plate is damaged by $50 \%$. Among them, Fig 4(a) is a three-dimensional view of the damage index, from which it can be clearly seen that unit 95 has damage; the damage location was further determined in Fig. 4(b). Because the four corners of square plate are fixed, the damage of four corner elements are not identified and analyzed in this paper. According to the above analysis, whether the damage is in the middle or edge of the plate, this index can identify the damage correctly.

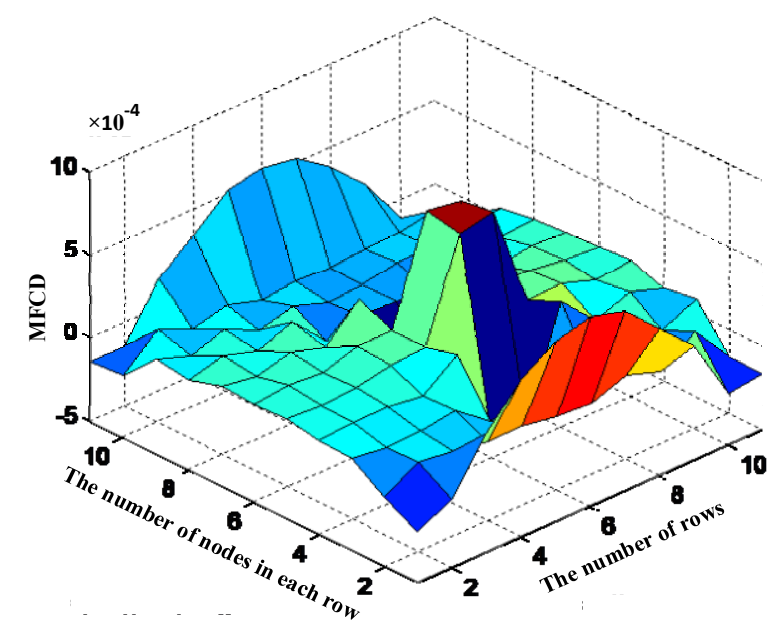

Fig. 2(a). Damage index of three-dimensional view.

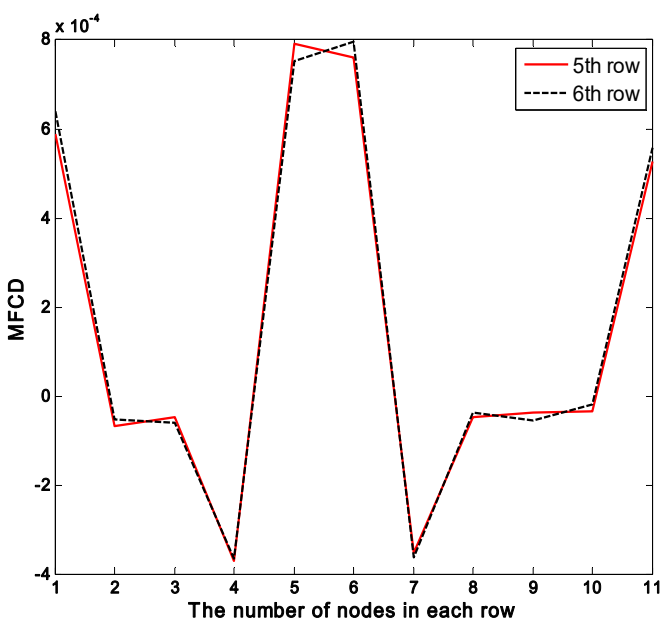

Fig.2(b). The 5th and 6th section curves.

Fig.2. The figure of the first order damage index in case 1.

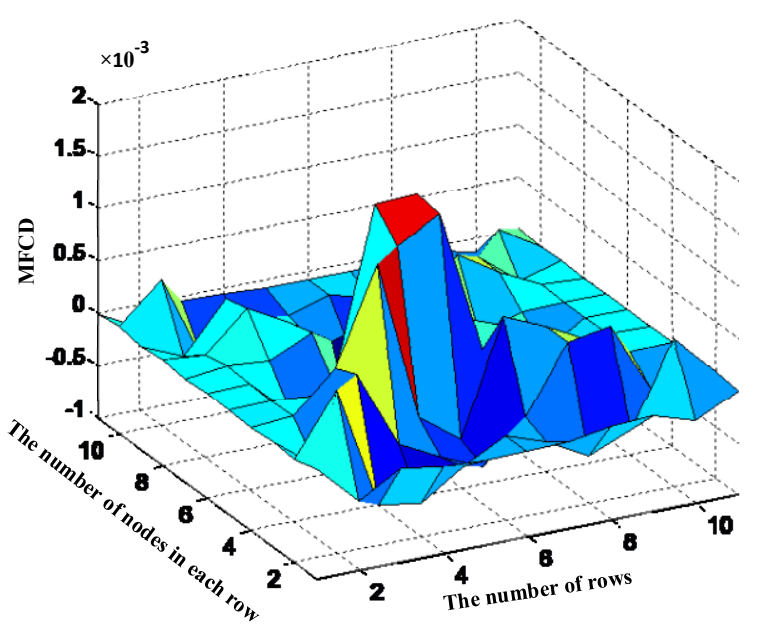

Fig. 3(a). Damage index of three-dimensional view.

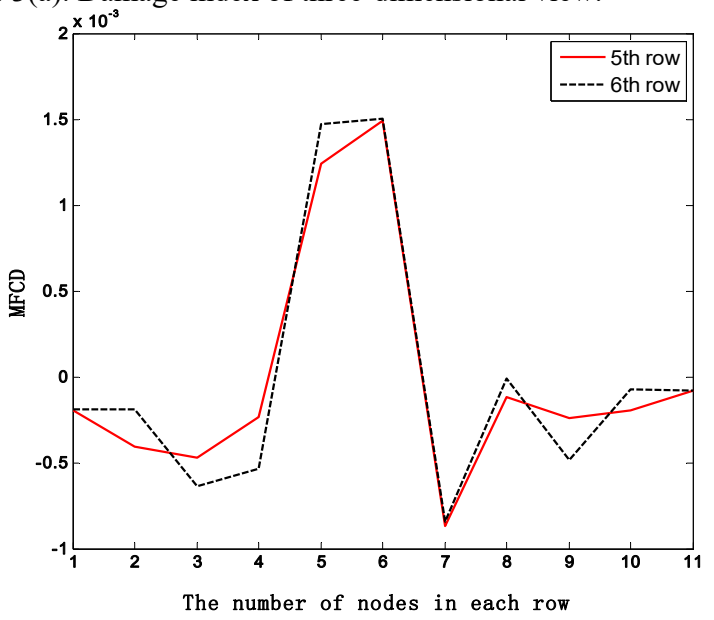

Fig. 3(b). The 5th and 6th section curves.

Fig. 3. The figure of the ahead 3-orders damage index in case 1. 


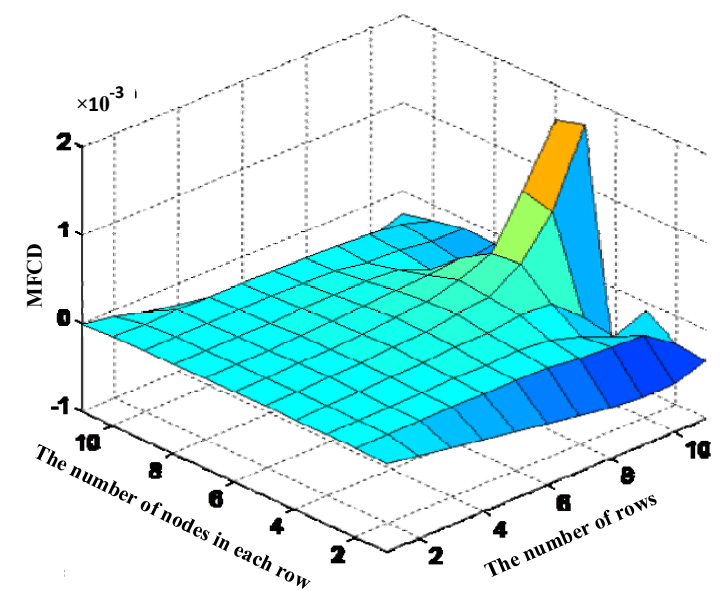

Fig. 4(a). Damage index of three-dimensional view.

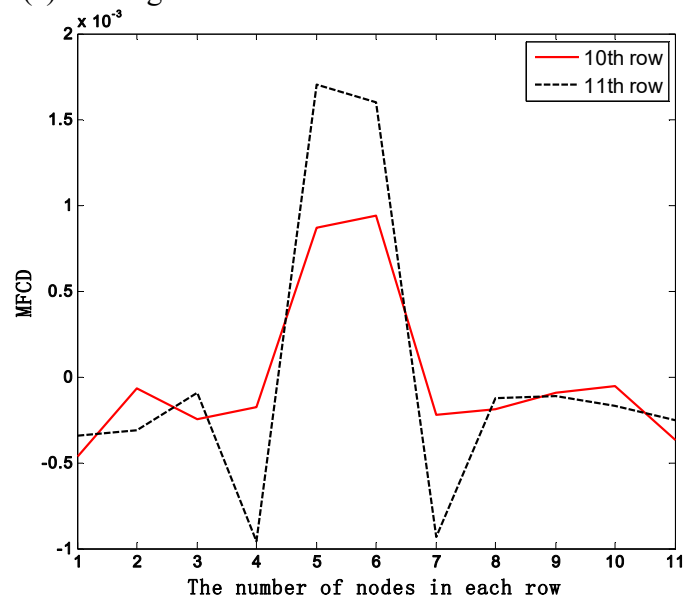

Fig. 4(b). The 10th and 11th section curves.

Fig. 4. The figure of the first order damage index in case 2.

\subsubsection{Multi-positions damage identification}

When multiple damage occurs in the structure, can the index accurately identify multiple damage locations? It is assumed that unit 45 and unit 95 both are damaged by $50 \%$ at the same time, the figure of damage index as shown in Fig. 5. Furthermore, it is supposed that unit 23 and unit 77 both are damaged by $50 \%$ at the same time, that is, the damage is located in different rows, and in different columns, the figure of damage index as shown in Fig. 6.

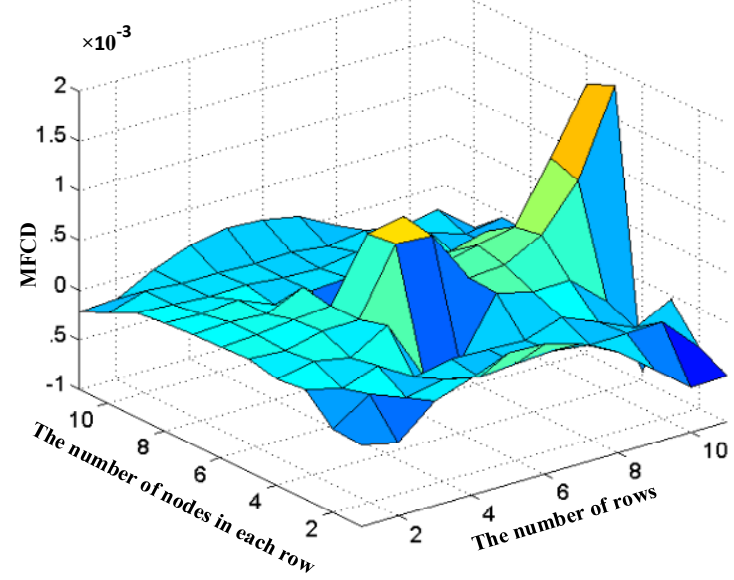

Fig. 5(a). Damage index of three-dimensional view.

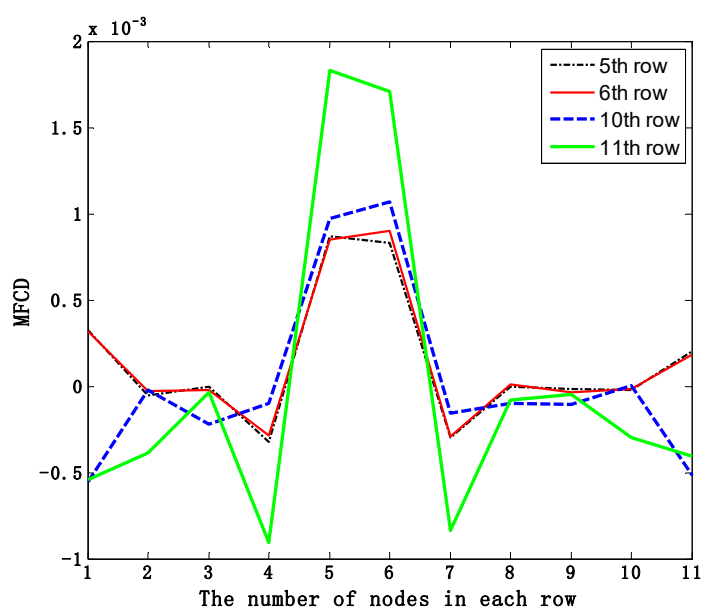

Fig. 5(b) .The 5th, 6th, 10thand11th section curves.

Fig. 5. The figure of the first order damage index in case 3 .

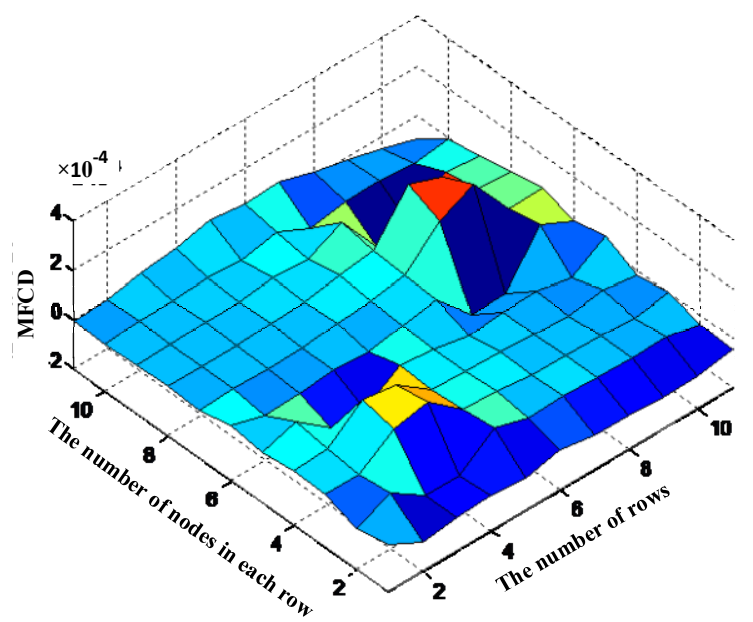

Fig. 6(a). Damage index of three-dimensional view.

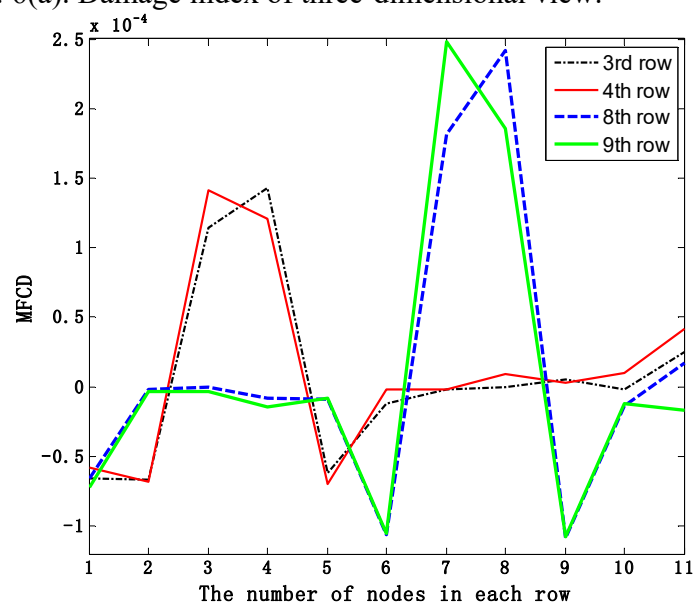

Fig. 6(b). The 3rd, 4th, 8th and 9th section curves.

Fig.6. The figure of the first order damage index in case 4 .

We can see from Fig.5 and Fig.6, for multiple injuries, wherever the damage is, the index can accurately identify them. However, when multiple injuries are of the same degree of damage, there are some differences in the recognition of damage degree. 


\subsubsection{Damage degree assessment}

The judgment of damage degree is mainly based on comparing the size of damage indexes that are at the same position of the structure and under different damage degree. On the premise of confirmed the damage location, in order to distinguish the damage size more clearly, we select one of the two section curves related to the damage element in the damage index diagram. It is assumed unit 45 is damaged by $20 \%, 50 \%$ and $70 \%$ respectively, the index size of the damage degree are shown in Fig. 7.

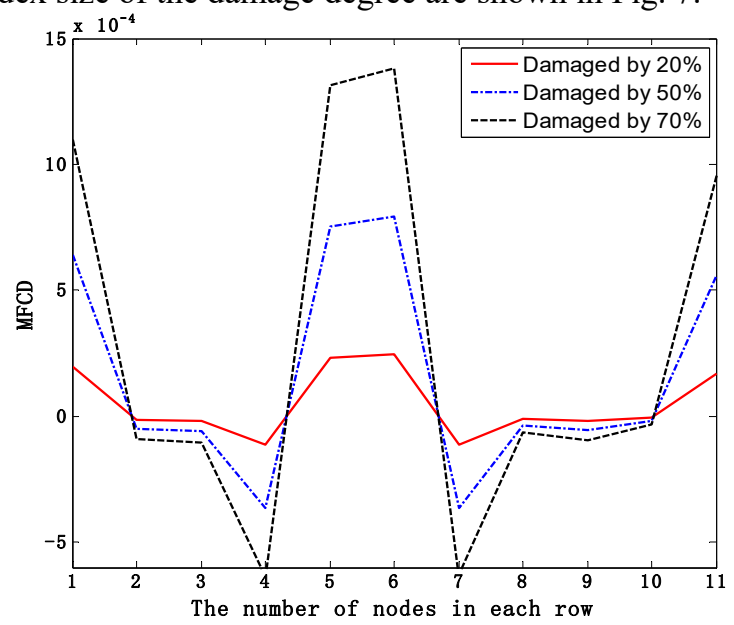

Fig. 7. The 6th row section curves of damage index figure in case 5 .

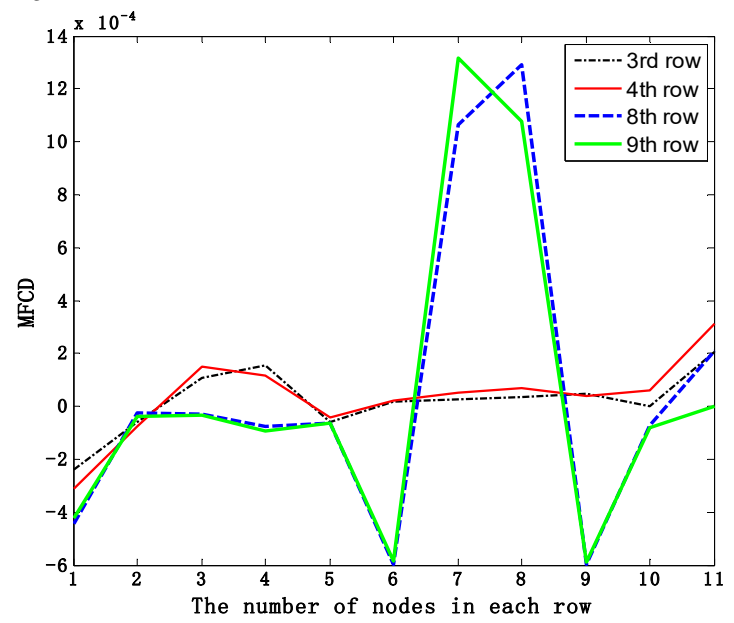

Fig. 8. The 3rd, 4th, 8th, 9th rows section curves of damage index figure in case 6 .

Fig. 7 is the $6^{\text {th }}$ row section curves in damage index graph that related to the damage element when unit 45 is damaged by $20 \%, 50 \%$, and $70 \%$ respectively. We can see from the Fig. 7 clearly, with the increase of damage degree, the value of damage index curve at the node of damage unit (that is node 5 and node 6) also increased significantly, this indicates that the index can reflect the size of a single damage degree. Fig. 8 is the $3^{\text {rd }}, 4^{\text {th }}, 8^{\text {th }}$, and $9^{\text {th }}$ rows section curves in damage index graph when unit 23 is damaged by $20 \%$, and unit 77 is damaged by $70 \%$ at the same time. A comparison between Fig. 8 and Fig. 6(b) shows that, when both unit 23 and unit77 are damaged by $20 \%$, the index value of unit 23 is about
$1.5 \times 10^{-4}$, and the index value of unit 77 is about $2.5 \times 10^{-4}$, there are not much difference; but when unit 23 is damaged by $20 \%$, and unit 77 is damaged by $70 \%$ at the same time, the index value of unit 23 is still about $1.5 \times 10^{-4}$, the damage index value of unit 77 is near $13 \times 10^{-4}$,the results are very different. Moreover, from the damage index values of two graphs, the index value of $(1.5 \square 2.5) \times 10^{-4}$ correspond to $20 \%$, and the index value of $13 \times 10^{-4}$ correspond to $70 \%$, this indicates that the index can fully reflect the size of damage degree. But we can know from Fig. 8, when the two degree of structure damage differ greatly, the small one is easy to be ignored, or we may confuse the single damage and multiple damage, because of the magnitude difference between the two damage indexes being large. For the damage identification index of longitudinal flexure curvature difference, the recognition result is the same as above, which is not repeated here.

\section{Conclusion}

It has been present that use the damage identification index of MFCD to identify the location of damage and determine the extent of damage. The plate had been divided by finite element method according to the theory of Kirchhof thin plate. In this article, the single-position damage and multi-position had been simulated and calculated with reducing the unit stiffness. We had found that the MFCD can not only accurately identify the single-damage and multi-damage position of plate structure, but also reflect the size of damage degree by analyzing. It has a great significance to apply the index into the range of damage identification of actual structures.

\section{References}

1. Doebling S W. Damage identification and health monitoring of structural and mechanical systems from changes in their vibration characteristics: a literature review[R]. Los Alamos National Laboratory Report LA-13070-MS, 1996.

2. Peeters B and Roeck G D. Preference-based stochastic subspace identification for output-only modal analysis[J]. Mechanical Systems and Signal Processing, 1999, 13: 855-878.

3. F. Seco and A. R. Jimenez. Modal analysis of the piezoelectric generation of ultrasonic guided waves for nondestructive testing of cylindrical structures[J]. Russian Journal of Nondestructive Testing, 2007(43):683-691.

4. S. N. Shevtsov, V. A. Akopyan, E. V. Rozhkov. An approach to the problem of damage identification in an elastic rod based on the Timoshenko beam model[J]. Russian Journal of Nondestructive Testing, 2011. 
5. Z Y Shi, S S Law, LM Zhang. Structural damage detection from modal strain energy change[J]. Journal of Engineering Mechanics, 2000 (12):1216-1223.

6. Fukunaga Hisao, Kameyama Masaki, Ogi Yoshiro. Damage identification of laminated composite structures based on dynamic residual forces[J]. Advanced Composite Materials, 2001(2):209-218.

7. Pandey A K. Biswas M. Damage detection in structures using changes in flexibility[J]. Journal of Sound and Vibration, 1994, 169(1): 3-17.

8. Pandey A K, Biswas M. Experimental verification of flexibility difference method for locating damage in structure[J].Journal of Sound and Vibration,1995, 184(2):311-328.

9. Hui Cao, Michael I. Fridewll. Damage detection method based on modal flexibility curvature[J]. Engineering Mechanics, 2006(23)4: 33-38.

10. Hui Cao, Xinliang Zhang, Yingmin Li. The damage identification of frame using modal flexibility curvature difference[J]. Vibration and Impact, 2007(26)6: 116-124.
11. Qiuwei Yang, Jike Liu. Research progress of flexibility method for damage identification of engineering structures[J]. Vibration and Impact, 2011(30)12: 147-153.

12. Dian $\mathrm{Xu}$, Youfa Yang. The identification method of damage structure based on flexibility curvature matrix difference $[\mathrm{J}]$. Journal of Shenyang University of Technology, 2012(34)6: 715-720.

13. Feili Qiu, Limin Zhang, Weihua Zhang. The identification of structure damage based on modal flexibility matrix[J]. Noise and Vibration Control, 2015(35)4: 101-106.

14. Xiaoyan Liu, Taixin Jiang, Guanghui Wang. The research of damage identification method based on modal flexibility matrix[J]. Journal of Changsha University, 2017(31)2: 15-19.

15. Li Chen. The research of structure damage identification based on flexibility curvature matrix[D]. Dalian University of Technology (Doctoral Thesis), 2009. 